\title{
AC conductivity measurements of ultradilute colloidal suspensions in HEPES buffer
}

Aditya Vikram, Khandelwal, Akash Singh, Namrata Pal, Rajdeep Kumar, Gaurav Goel* and Shalini Gupta*

Dept. of Chemical Engineering, Indian Institute of Technology Delhi, 110016, India

*Corresponding authors: shalinig@ chemical.iitd.ac.in and goelg@chemical.iitd.ac.in

\section{Supplementary Information (SI)}
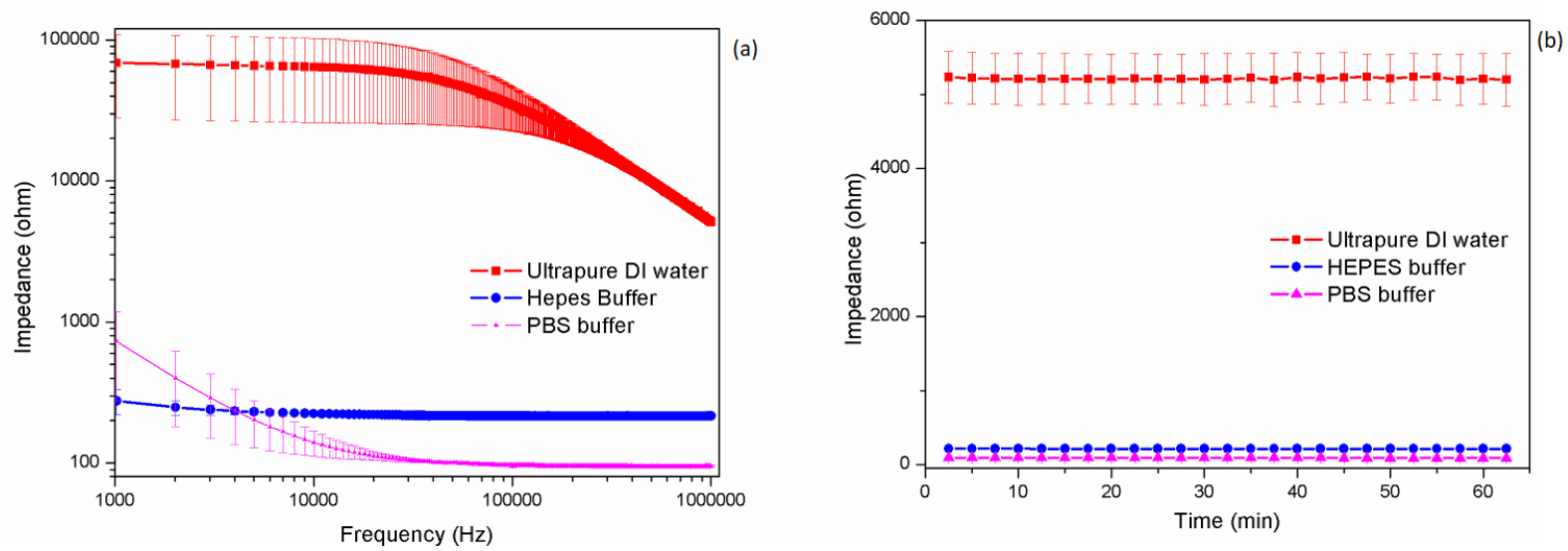

Fig. S1. Impedance spectra as a function of (a) frequency and (b) time in different media. The results in (a) are reported $5 \mathrm{~min}$ after sample loading. Here, the flatness of the HEPES curve unlike water and PBS is suggestive of negligible electrode polarization effects. In (b), the results are reported at $f=1 \mathrm{MHz}$.

\section{Calculation of working frequency range}

Chassagne et al. [1] have given expressions for the frequency up to which mobile charges lead to full build-up of double layer at blocking electrodes $\left(\omega_{P}\right)$ and the frequency above which the effect of electrode polarization can be completely ignored $\left(\omega_{b}\right)$. The two expressions are given as per equations $\mathrm{S} 1$ and $\mathrm{S} 2$ :

$$
\omega_{P}=2 \pi f_{P}=\frac{2 \kappa a D_{0}}{d a}
$$




$$
\omega_{b}=2 \pi f_{b}=\sqrt{\omega_{0} \omega_{P}} ; \omega_{0}=D_{0} \kappa^{2}
$$

where, $D_{0}$ is diffusivity of ion, $\kappa^{-1}$ is Debye length, $a$ is particle radius, and $d$ is electrode gap.

The values of various parameters used to calculate $f_{P}$ and $f_{b}$ for a suspension of PS particles in PBS buffer are as below:

\begin{tabular}{|l|l|lrl|ll|l|}
\hline $\begin{array}{l}\text { Parameter } \\
\text { values: }\end{array}$ & $d, \mathrm{~m} \quad 1.00 \mathrm{E}-04$ & $a, \mathrm{~m}$ & $5.00 \mathrm{E}-07$ & $\kappa a$ & $7.04 \mathrm{E}+02$ & $D_{0}, \frac{m^{2}}{s}$ & $2.00 \mathrm{E}-09$ \\
\hline $\begin{array}{l}\text { Electrode polarization frequencies for a suspension of PS } \\
\text { particles in PBS buffer }\end{array}$ & $\omega_{P}, \frac{1}{s}$ & $5.63 \mathrm{E}+04$ & $\omega_{b}, \frac{1}{s}$ & $1.49 \mathrm{E}+07$ \\
\cline { 2 - 6 } & $f_{P}, \frac{1}{s}$ & $8.96 \mathrm{E}+03$ & $f_{b}, \frac{1}{s}$ & $2.38 \mathrm{E}+06$ \\
\hline
\end{tabular}

(a)

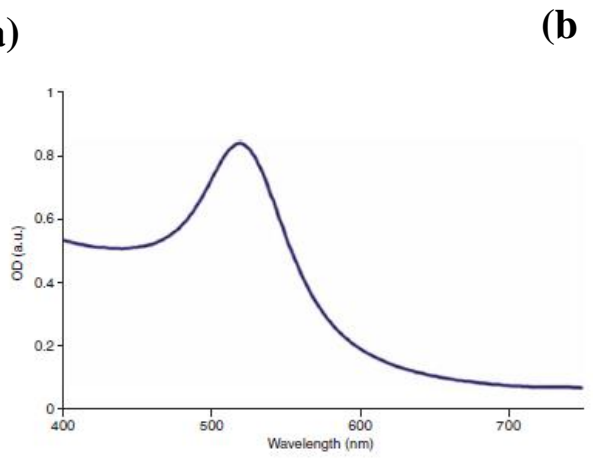

(b

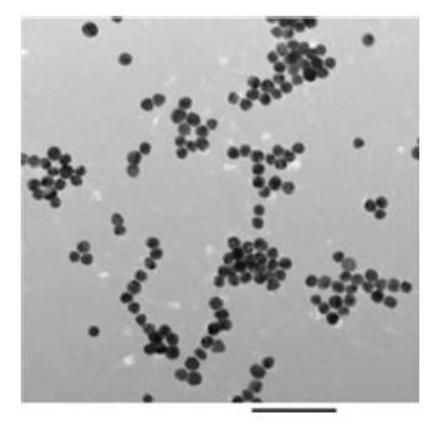

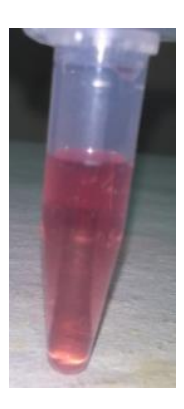

Fig. S2. Characterization of Au NPs: (a) UV-Visible spectrum, (b) TEM image and, (c) actual digital camera image of an Au NP suspension showing monodisperse particles of size $16 \pm 4 \mathrm{~nm}$. The scale bar is $100 \mathrm{~nm}$.

\section{Preparation of metallodielectric particles}

The Au NPs were prepared as discussed in the main text and characterized as shown below (Fig. S2). Following this, $20 \mu \mathrm{L}$ of $2 \%$ PS suspension was co-incubated with $1 \mathrm{~mL}$ of $1.12 \mathrm{nM} \mathrm{Au} \mathrm{NP}$ suspension. As the time of incubation was increased from $4 \mathrm{~h}$ to $24 \mathrm{~h}$, the number of Au NPs attached to the PS beads also increased, or conversely, the number of Au NPs remaining in the supernatant decreased (Fig. S3a). To convert the UV-visible spectra into Au NP concentration, we used the following ref:[2]. 


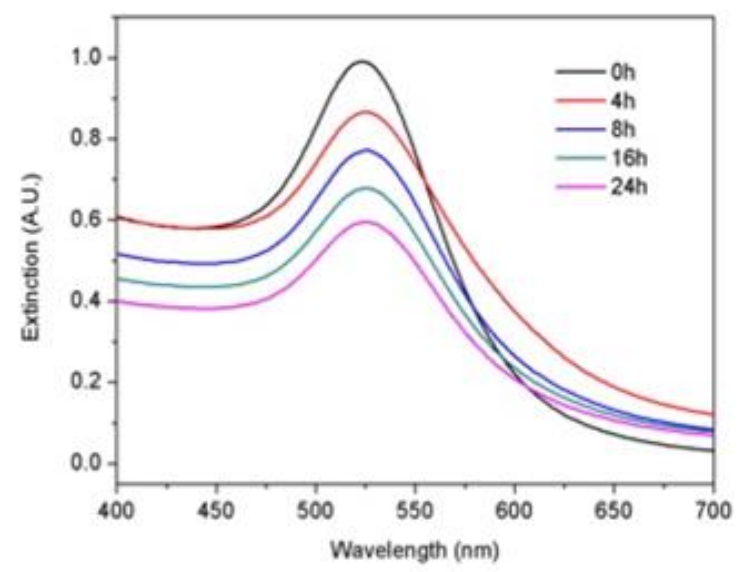

Fig. S3. (a) UV-visible spectra illustrating the concentration of the Au NPs remaining in the supernatant after binding to the PS beads.

0 min

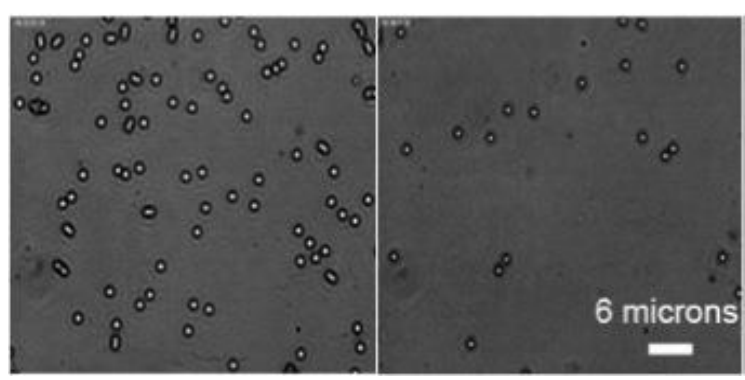

$3 \mathrm{~min}$

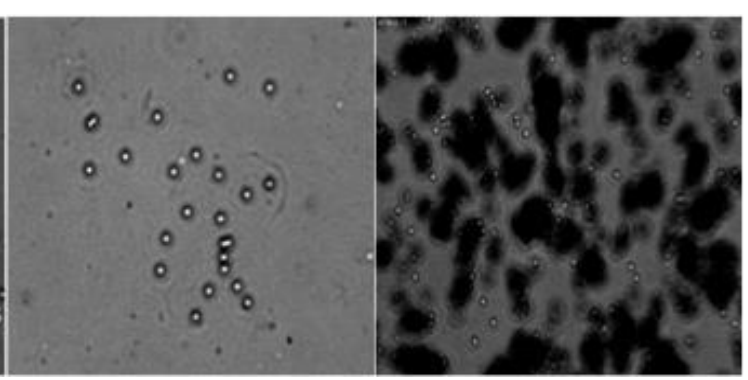

Fig. S4. Time-lapsed optical micrographs of Au-PS particles with increasing durations of silver enhancement. The $2 \%$ w/v PS beads were coated with Au NPs via $24 \mathrm{~h}$ of incubation process as discussed above. The silver enhancement procedure followed was as per the user manual. All the images were taken using a 100× oil immersion objective mounted on a BX53 optical microscope using a CCD camera (Pixelfly, PCO, Germany). Although the extent of silver coverage on the $\mathrm{Au}$ NPs was not quantified, it was observed that the Ag-Au-PS particles had moderate colloidal stability. They precipitated noticeably when stored at $4{ }^{\circ} \mathrm{C}$ beyond a day. The process, however, was reversible as the precipitates could be redispersed via ultrasonication. 


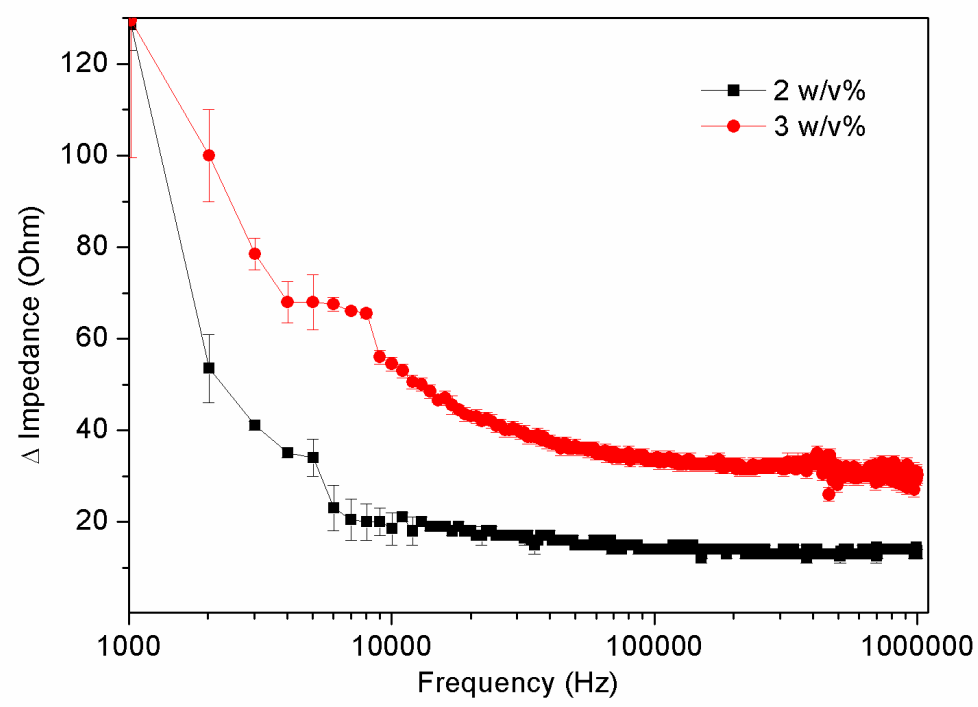

Fig. S5. Experimental values of the dielectric increment as a function of frequency for 2 and 3 w/v \% PS particles in HEPES buffer. The graph clearly shows alpha relaxations obtained around 5 and $8 \mathrm{kHz}$, for 2 and $3 \mathrm{w} / \mathrm{v} \%$ particles, respectively.

\section{Permittivity and conductivity increment calculations}

The complex conductivity $\left(K^{*}(\omega)\right)$ of the system was obtained from the experimentally measured impedance values $(Z(\omega))$ using the relation [3] (eq. 1)

$$
K^{*}(\omega)=\frac{1}{C_{c} Z_{s}(\omega)}
$$

where, $C_{c}$ is the electrode cell constant. It was obtained using the relation, $C_{c}=K Z$, where, $K=$ $1.29 \mathrm{~S} / \mathrm{m}$ was obtained by measuring the conductivty of a standard $0.1 \mathrm{M} \mathrm{KCl}$ solution at $25^{\circ} \mathrm{C}$ on our chip and $Z=119 \Omega$ was obtained by measuring the impedance of the same $\mathrm{KCl}$ solution at $25{ }^{\circ} \mathrm{C}$ on our chip. By doing this, the value of $C_{c}$ was obtained as $153.5 \mathrm{~m}^{-1}$. The real part of suspension conductivity $\left(K^{\prime}{ }_{s}(\omega)\right)$, which is a measure of resistance in the system, is related to complex conductivity as (eq. 2),

$$
K_{s}^{*}(\omega)=K_{s}^{\prime}(\omega)+i \omega \varepsilon_{o} \varepsilon_{s}^{\prime}(\omega)
$$


where, $\varepsilon^{\prime}{ }_{S}(\omega)$, the real is part of complex permittivity, is a measure of capacitance in the system. For the buffer alone, this quantity reduces to eq. $\mathbf{3}$,

$$
K_{e}^{*}(\omega)=K_{e}+i \omega \varepsilon_{o} \varepsilon_{e}
$$

where, $K_{e}$ is the DC conductivity of the electrolyte and $\varepsilon_{e}$ is the relative dielectric permittivity of the electrolyte solution independent of frequency. The complex permittivity is related to complex conductivity as (eq. 4),

$$
\varepsilon_{S}^{*}(\omega)=\frac{K_{S}^{*}(\omega)}{i \omega \varepsilon_{o}}
$$

Under the assumption of low concentration or, no particle-particle interactions, the change in complex conductivity or complex permittivity due to a single particle is calculated as per eq. $\mathbf{5} \boldsymbol{\&}$ 6, respectively.

$$
\begin{aligned}
\frac{\Delta K_{s}^{*}}{\emptyset} & =\frac{K_{S}^{*}(\omega)-K_{e}^{*}(\omega)}{\emptyset} \\
\frac{\Delta \varepsilon_{S}^{*}}{\emptyset} & =\frac{\varepsilon_{S}^{*}(\omega)-\varepsilon_{e}^{*}(\omega)}{\emptyset}
\end{aligned}
$$

where, $\emptyset$ is the volume fraction of particles in the suspension.

\section{O'Brien's analytical solution}

In the limit of thin double layer, O'Brien et al. [4] gave the measure of the dipole moment generated by the polarization of the particle core and its double layer as,

$$
C_{0}(\omega)=\frac{\left(2 \lambda-\frac{i \omega \varepsilon_{i n t}}{K_{e}}\right)-\left(1-\frac{i \omega \varepsilon_{O}}{K_{e}}\right)}{2\left(1-\frac{i \omega \varepsilon_{0}}{K_{e}}\right)+\left(2 \lambda-\frac{i \omega \varepsilon_{i n t}}{K_{e}}\right)}
$$

where, $C_{0}(\omega)$ is the dipole-coefficient and $\varepsilon_{\text {int }}$ is the relative permittivity of particle core. The complex permittivity increment $\Delta K_{S}^{*}(\omega)$, on ignoring $\mathrm{O}\left(\phi^{2}\right)$ and higher terms, is given by, 


$$
\frac{\Delta K_{s}^{*}(\omega)}{\phi}=\frac{3}{a^{3}}\left(K_{e}-i \omega \varepsilon_{0}\right) C_{0}(\omega)
$$

To compare this theoretical prediction with experimental data, we subtract a frequency independent offset from only the real part of $\Delta K_{s}^{*}(\omega)$, as was done by Beltramo et al. [5] The expression for double layer conductivity parameter $\lambda$ was taken from equation 17 of Mangelsdorf et al.[3] when $\exp \left(-\frac{e z_{i} \xi}{k_{b} T}\right) \gg 1$ and equation 21 (reproduced below) when this condition is not satisfied. Assuming ions with equivalent mobility (or, diffusivity), the expression for double layer conductivity parameter $\lambda$ is given by

$$
\begin{gathered}
\lambda=\frac{2}{\mathrm{\kappa a}}\left(1+\frac{3 m}{\mathrm{z}^{2}}\right)\left[\cosh \left(\frac{\mathrm{ez} \xi}{2 \mathrm{kBT}}\right)-1\right] \\
\mathrm{m}=\frac{z_{i} \lambda_{\text {avg } \mu}}{\mathrm{e}} \\
\mu=\frac{\varepsilon_{o} \mathrm{k}_{\mathrm{B}} \mathrm{T}}{6 \pi \eta_{\mathrm{o}} \mathrm{e}}
\end{gathered}
$$

where, $\lambda_{\text {avg }}$ is the average drag coefficient calculated from a composition weighted average of individual drag coefficients, $\lambda_{i}$, given by,

$$
\lambda_{i}=\frac{k_{b} T}{D_{i}}
$$

where $D_{i}$ is diffusivity of ion.

\begin{tabular}{|c|c|}
\hline Ion & Ionic diffusivity, $\mathbf{~ m}^{\mathbf{2}} \mathbf{s}$ \\
\hline $\mathrm{Na}^{+}$ & $1.33 \times 10^{-9}$ \\
\hline HEPES ion & $5.00 \times 10^{-10}$ \\
\hline $\mathrm{K}^{+}$ & $1.96 \times 10^{-9}$ \\
\hline $\mathrm{Cl}^{-}$ & $2.03 \times 10^{-9}$ \\
\hline $\mathrm{PO}_{4}{ }^{3-}$ & $0.61 \times 10^{-9}$ \\
\hline
\end{tabular}




\section{Calculation of the correction factor for permittivity increment}

For frequencies between $\omega_{p}<\omega<\omega_{b}$ electrode polarization (EP) effects are important such that one needs to compensate for EP effects to correctly calculate the real part of suspension permittivity $\varepsilon^{\prime}{ }_{S}(\omega)$ in the range $\left[\omega_{p}, \omega_{b}\right]$. To this end, we have used the approach given in section 5.1 of Chassagne et al. [1].

The impedance of the cell filled with suspension $Z_{c, s}^{*}$ has an EP contribution $Z_{E P, s}^{*}\left(=\frac{1}{i w C_{E P, S}}\right)$ and the contribution of the bulk of the suspension $Z_{s}^{*}$, latter being the quantity of interest. The impedenace of the cell is therefore given by:

$$
Z_{c, s}^{*}=Z_{s}^{*}+Z_{E P, s}^{*}
$$

The general relation between impedance and conductivity will be same as given in equation 1 (for any subscript: $x=\{c, s\},\{s\},\{c, e\},\{e\})$ :

$$
1 / Z_{x}^{*}=C_{c} K_{x}^{*}=C_{c} i \omega \varepsilon_{o} \varepsilon_{x}^{*}=C_{c}\left(K_{x}^{\prime}(\omega)+i \omega \varepsilon_{o} \varepsilon_{x}^{\prime}(\omega)\right)
$$

Since the capacitance at the electrode $\left(C_{E P, x}\right)$ can be different for the cell filled with the suspension and the cell filled with only the electrolyte, $Z_{E P, x}^{*}$ will not cancel out when using the subtraction method. This only affects the calculation of real part of permittivity. We have therefore used the following expression for calculating the real part of permittivity increment in the range $\left[\omega_{p}, \omega_{b}\right]$ (for details see equation 45, 50, 56 in ref. [1]):

$$
\Delta \varepsilon_{s}^{\prime}(\omega)=\varepsilon_{s}^{\prime}(\omega)-\varepsilon_{e}^{\prime}(\omega)=\varepsilon_{c, s}^{\prime}(\omega)-\varepsilon_{c, e}^{\prime}(\omega)-\left[\frac{\left(K_{S}\right)^{2}-\left(K_{e}\right)^{2}}{\left(\omega \varepsilon_{o}\right)^{2} \varepsilon_{e} \kappa d / 2}\right]
$$

where, $\kappa$ is the Debye length of electrolyte and $d$ is electrode spacing. The term in the square brackets is the correction term for determination of permittivity increment from impedance measurements on a cell filled with either the suspension or just the electrolyte. 


\section{Calculation of Debye lengths $\left(\kappa^{-1}\right)$ in PBS and HEPES buffer}

The Debye length was obtained from the relation given by Debye-Huckel theory [6],

$$
\kappa^{2}=\sum_{1}^{n} \frac{e^{2} z_{j}^{2} n_{j}^{\infty}}{k_{b} T}
$$

where, $e$ represents the charge of an electron, $z_{j}$ stands for the charge on $\mathrm{j}^{\text {th }}$ ionic species, $n_{j}^{\infty}$ is the ionic density for the species far away from the particle, $k_{b}$ is the Boltzmann constant. $n_{j}^{\infty}$ was obtained from the respective concentration of the ionic species.

The concentration of anionic HEPES $\left(A^{-}\right)$in $40 \mathrm{mM}$ HEPES buffer at $\mathrm{pH} 8.5$ was obtained from the Henderson-Hasselbach equation:

$$
\begin{gathered}
p H=p K a+\log \frac{\left[A^{-}\right]}{[H A]} \\
8.5=7.5+\log \frac{\left[A^{-}\right]}{[H A]}
\end{gathered}
$$

$\left[A^{-}\right]=10[H A]=10\left[40-\left[A^{-}\right]\right]=36.36 \mathrm{mM}$.

The other ionic concentrations are

$$
\begin{aligned}
& {\left[\mathrm{H}^{+}\right]=10^{-8.5}=3.16 \mathrm{nM}} \\
& {\left[\mathrm{OH}^{-}\right]=10^{-5.5}=3.16 \mu \mathrm{M}} \\
& {\left[\mathrm{Na}^{+}\right]=40 \mathrm{mM}}
\end{aligned}
$$

Similar steps were repeated for $150 \mathrm{mM}$ PBS at pH 7.4 which has the composition $137 \mathrm{mM} \mathrm{Na}^{+}$, $2.7 \mathrm{mM} \mathrm{K}^{+}, 139 \mathrm{mM} \mathrm{Cl}^{-}, 10 \mathrm{mM} \mathrm{PO}_{4}{ }^{3-}$. The final values obtained are reported in Table $\mathbf{S 1}$.

Table S1: The values of our system parameters.

\begin{tabular}{|c|c|c|}
\hline Suspension type & Zeta potential $(\boldsymbol{\zeta}), \mathbf{m V}$ & ка \\
\hline PS in HEPES & -65 & 327.74 \\
\hline PS in 1x PBS & -39.5 & 703.59 \\
\hline Au NP in HEPES & -26.3 & 5.24 \\
\hline
\end{tabular}




\section{Calculations for amount of Tween 20 added to fully saturate a particle suspension}

For a $10 \mu \mathrm{L} 0.1 \%$ v/v PS suspension, the total volume of particles in the system,

$$
\begin{aligned}
\frac{4}{3} \pi r_{p}^{3} N_{p} & =\frac{0.1 \times 10 \mu l}{100}=0.01 \mu l \\
\text { Or, } N_{p} & =\frac{0.01}{\frac{4}{3} \pi r_{p}^{3}}
\end{aligned}
$$

where, $N_{p}$ is the total number of particles. So, the total surface area available for surfactant adsorption $=4 \pi r_{p}^{2} N_{p}=\frac{0.03 \times 10^{-9}}{0.5 \times 10^{-6}}=6 \times 10^{-5} \mathrm{~m}^{2}$. Now, the cross-sectional area of one Tween 20 molecule $\sim 133 \mathrm{~A}^{o 2}$.[7] Therefore, the total concentration of Tween 20 required is

$$
\left(\frac{6 \times 10^{-5} \mathrm{~m}^{2}}{4 \pi(66.5)^{2}}\right) \times\left(\frac{1}{10 \times 10^{-6} L}\right)=7.5 \mu \mathrm{M} \text { or } 0.017 \mathrm{v} / \mathrm{v} \% \text {. }
$$

Since this surfactant amount corresponds to the saturation level in the highest particle concentration suspensions used, we kept this concentration fixed in all our surfactant-based experiments. This meant there would be excess unbound Tween 20 present in solution in all the lower particle concentration cases. To account for the impedance variation due to these remaining molecules, impedance data for all lower concentration suspensions were reported after subtraction of the appropriate baseline, i.e., 7.5 $\mu \mathrm{M}-\mathrm{M}_{\text {lower, }}$ where, $\mathrm{M}_{\text {lower }}$ is the saturation concentration for a particular lower concentration (calculated using the same approach as above).
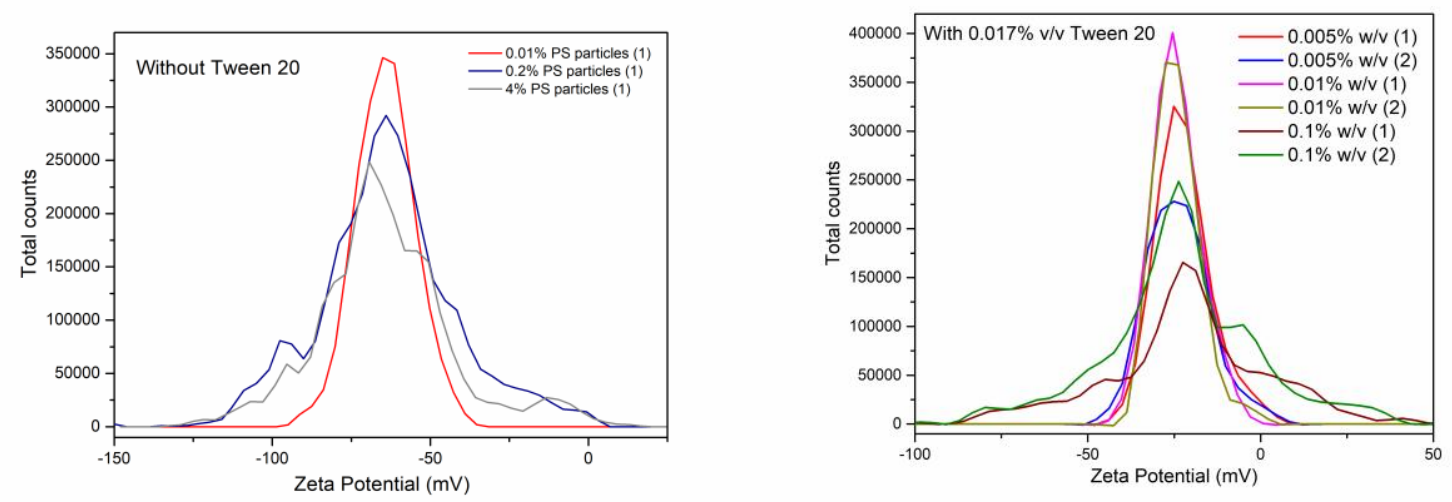

Fig. S6. Zeta potential values of PS particles before and after Tween 20 addition. 


\section{Why should HEPES give better S/N ratio?}

The reason why HEPES gives higher $\mathrm{S} / \mathrm{N}$ ratio is believed to be linked to the ionic size asymmetry of the HEPES molecule as other zwitterionic molecules in the same class also gave us a similar response (unpublished data). Below we report a graph that shows how the $\mathrm{S} / \mathrm{N}$ ratio for HEPES improves as we increase the $\mathrm{pH}$ in a $0.1 \% \mathrm{w} / \mathrm{wv}$ PS suspension from 5.5 to 8.5. This is true in general for dilute suspensions that are on the whole more conductive than buffer alone ( $\triangle \mathrm{Z}$ negative). Since HEPES exists as a completely ionized hybrid molecule with no resultant charge (or in other words, a zwitterion) at $\mathrm{pH} \sim 5.5$ and as a negatively charged molecule (or in words, a salt) at $\mathrm{pH}>7$, we believe that it is the addition of salt that improves the $\mathrm{S} / \mathrm{N}$ ratio. But why this improvement is 3-4 times better with HEPES as compared to PBS is still not clear.

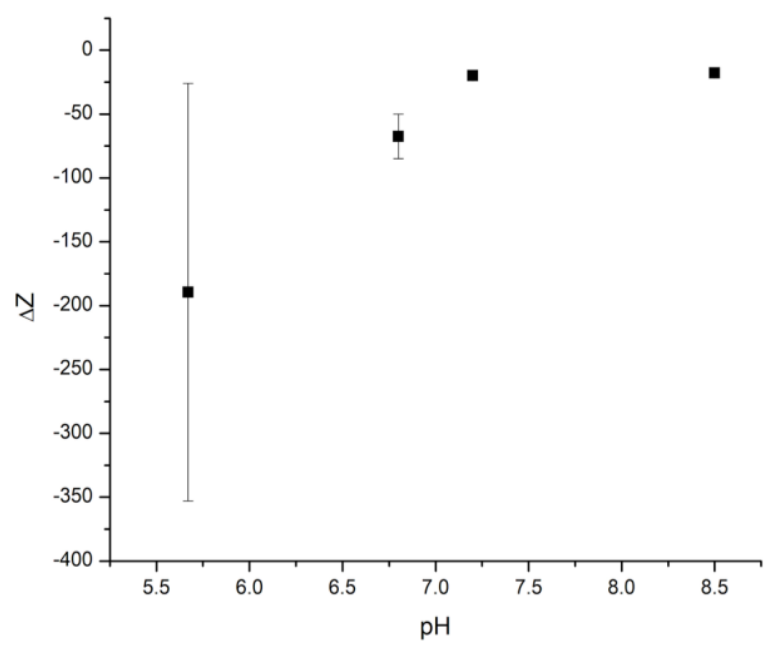


(a)
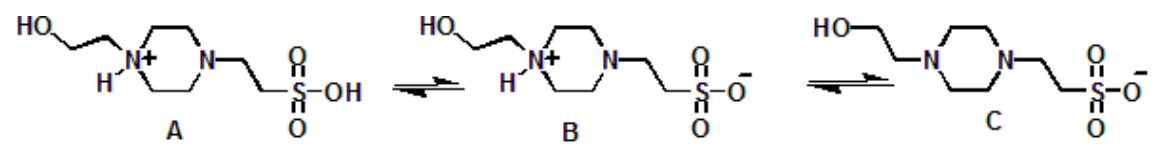

(b)

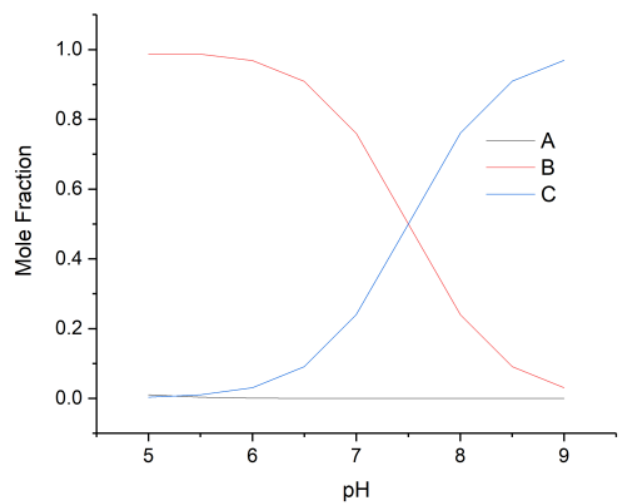

Fig. S7. (a) The different states of HEPES molecule having pKa values at 3 and 7.5, respectively. (b) The graph shows the calculated relative mole fraction of each state as a function of $\mathrm{pH}$. 
(a)
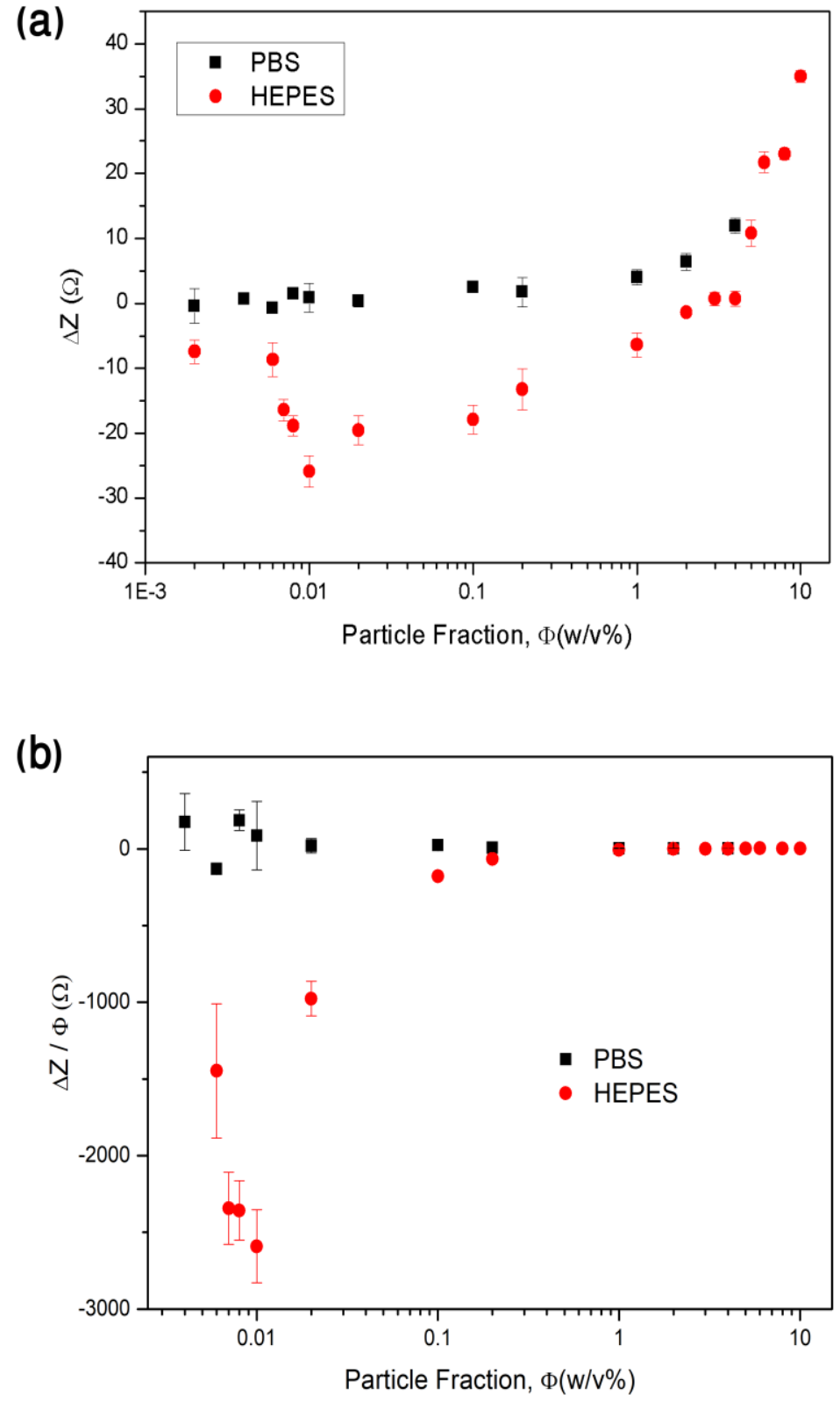

Fig. S8. Comparison of impedance response between conductivity matched PBS and HEPES buffers at $0.2 \mathrm{~S} / \mathrm{m}$ : (a) shows total $\Delta \mathrm{Z}$ response and (b) shows specific $\Delta \mathrm{Z}$ response as a function of particle fraction. The PBS concentration used was $14.92 \mathrm{mM}(\mathrm{pH} 7.36)$ and HEPES buffer was the same as that used in the main text (i.e., $40 \mathrm{mM}$ at $\mathrm{pH} 8.5$ ). The absolute experimental data values are slightly different from those in the main text as these data have been procured using $100 \mu \mathrm{m}$ gap IDEs with a slightly different cell constant as well as with $1 \mu \mathrm{m}$ PS particles from a different lot but same manufacturer. 


\section{$\underline{\text { Calculation for electrokinetic length scale for particle-particle interaction }}$}

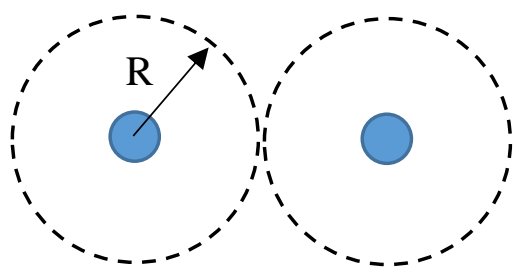

$\Phi_{w / v}=\left(\frac{\left(\frac{4}{3}\right) \pi a^{3}}{\left(\frac{4}{3}\right) \pi R^{3}}\right) \times f$ where, $\mathrm{f}=0.63$ is the packing factor for randomly packed hard spheres.

Since, $a=0.5 \mu \mathrm{m}$ and $\Phi_{w / v}=0.007 \%$, value of $\mathrm{R}$ obtained is equal to $10.4 \mu \mathrm{m}$. Therefore, interparticle distance $=2 \mathrm{R}=20.8 \mu \mathrm{m}$.

\section{References:}

[1] C. Chassagne, E. Dubois, M.L. Jiménez, J.P.M. van der Ploeg, J. van Turnhout, Compensating for Electrode Polarization in Dielectric Spectroscopy Studies of Colloidal Suspensions: Theoretical Assessment of Existing Methods, Frontiers in Chemistry. 4 (2016) 1-19. doi:10.3389/fchem.2016.00030.

[2] X. Liu, M. Atwater, J. Wang, Q. Huo, Extinction coefficient of gold nanoparticles with different sizes and different capping ligands, Colloids and Surfaces B: Biointerfaces. 58 (2007) 3-7. doi:10.1016/j.colsurfb.2006.08.005.

[3] C.S. Mangelsdorf, L.R. White, Dielectric response of a dilute suspension of spherical colloidal particles to an oscillating electric field, Journal of the Chemical Society, Faraday Transactions. 93 (1997) 3145-3154. doi:10.1039/a701289f.

[4] R.W. O’Brien, The high-frequency dielectric dispersion of a colloid, Journal of Colloid And Interface Science. 113 (1986) 81-93. doi:10.1016/0021-9797(86)90208-0.

[5] P.J. Beltramo, E.M. Furst, Transition from Dilute to Concentrated Electrokinetic Behavior in the Dielectric Spectra of a Colloidal Suspension, Langmuir. 28 (2012) 10703-10712. doi:10.1021/la301876w.

[6] J.N. Israelachvili, Intermolecular and Surface Forces: Third Edition, 2011. doi:10.1016/C2011-0-05119-0.

[7] https://pubchem.ncbi.nlm.nih.gov/compound/Tween_20\#section=Chemical-and-Physical$\underline{\text { Properties }}$ 